\title{
Prediction of Patient Outcome from Acinetobacter baumannii Bacteremia with Sequential Organ Failure Assessment (SOFA) and Acute Physiology and Chronic Health Evaluation (APACHE) II Scores
}

\author{
Su-Jung Chen ${ }^{1,2}$, Tze-Fan Chao ${ }^{3}$, Mei-Chun Chiang ${ }^{2}$, Shu-Chen Kuo ${ }^{1,2,5}$, Liang-Yu Chen ${ }^{2}$, \\ Ti Yin ${ }^{4}$, Te-Li Chen ${ }^{2,5}$ and Chang-Phone Fung ${ }^{2,5}$
}

\begin{abstract}
Objective Acinetobacter baumannii is an important nosocomial pathogen associated with a high mortality rate. However, no objective and quantitative severity scores are available for the severity stratification. We aimed to assess the effectiveness of SOFA and APACHE II scores calculated at the onset of bacteremia in predicting the mortality of patients with A. baumannii bacteraemia.

Patients and Methods A total of 110 patients with A. baumannii bacteremia were included in this retrospective study during the 40-month study period. Information including clinical and laboratory data was collected.

Results Multivariate analysis showed that both SOFA and APACHE II scores were independent outcome predictors after adjustment for other parameters. Goodness-of-fit was good for SOFA and APACHE II, and both models displayed excellent AUROCs (SOFA: $0.83 \pm 0.06$, APACHE II: $0.82 \pm 0.08$ in predicting 14-day mortality; SOFA: $0.85 \pm 0.04$, APACHE II: $0.81 \pm 0.04$ in predicting in-hospital mortality). There was no significant difference in the predictions of the two scoring systems, and the scores were highly correlated $\left(r^{2}=0.724, p<0.001\right)$. We found that SOFA $>8$, APACHE II $>29$ and SOFA $>7$, APACHE II $>23$ are associated with significantly higher 14-day and in-hospital mortality rates, respectively.

Conclusion SOFA and APACHE II scores assessed at the onset of bacteremia are reliable risk stratifying tools in predicting 14-day and in-hospital mortality in A. baumannii bacteremia. For ease of calculation, the use of SOFA rather than APACHE II score to predict mortality of A. baumannii bacteremia might have clinical application.
\end{abstract}

Key words: SOFA score, APACHE score, Acinetobacter baumannii, bacteremia, outcome

(Intern Med 50: 871-877, 2011)

(DOI: 10.2169/internalmedicine.50.4312)

\section{Introduction}

Acinetobacter baumannii is a significant cause of nosocomial infections and is associated with a high mortality rate (1), ranging from 17 to $63 \%$. Bacterial factors, such as the strain or the presence of certain virulence factors, may be associated with the outcome in patients with A. baumannii bacteremia. However, the virulence factors of A. baumannii are largely unknown (2). Other factors independently

\footnotetext{
${ }^{1}$ Department of Medicine, National Yang-Ming University Hospital, Taiwan, ${ }^{2}$ Division of Infectious Diseases, Department of Medicine, Taipei Veterans General Hospital, Taiwan, ${ }^{3}$ Division of Cardiology, Department of Medicine, Taipei Veterans General Hospital, Taiwan, ${ }^{4}$ School of Nursing, National Yang-Ming University, Taiwan and ${ }^{5}$ School of Medicine, Institute of Clinical Medicine, National Yang-Ming University, Taiwan

Received for publication August 2, 2010; Accepted for publication December 20, 2010

Correspondence to Dr. Te-Li Chen, tlchen@vghtpe.gov.tw
} 
associated with a poor prognosis of $A$. baumannii bacteremia include the severity of underlying diseases, pneumonia as the source of bacteremia, the presence of septic shock, disseminated intravascular coagulation, mechanical ventilator use, and inappropriate antibiotic therapy $(3,8)$. Although these prognostic factors play important roles in predicting clinical outcome, objective and quantitative severity assessment is more important with regard to therapeutic decision making. Such decisions compare the benefits of various treatments in different institutes, guide resource allocation, facilitate the description of illness severity, and allow explanation of patient condition to family members.

Several organ dysfunction scores have been developed to assess critically ill patients (9-11). The Acute Physiology and Chronic Health Evaluation II (APACHE II) and Sequential Organ Failure Assessment (SOFA) are two of the most commonly used organ dysfunction scoring systems. Although these were originally designed to classify and quantify the degree of organ failure, rather than predict patient outcome, several studies have demonstrated a clear relationship between organ dysfunction and mortality (9, 12-16).

Many intensive care units (ICUs) have used the APACHE II score, introduced in 1985 (17), to stratify critically ill patients according to risk (18-20). Although newer versions of the APACHE II prognostic model have been proposed (21), the original APACHE II model is still commonly used in many ICUs worldwide for auditing and research purposes. The Sepsis-related Organ Failure Assessment (9), a recently developed score, describes the degree of organ dysfunction over time and estimates the morbidity of ICU patients with sepsis (13). Later, it was realized that SOFA could also be applied to non-septic patients, the acronym "SOFA" was taken to refer to Sequential Organ Failure Assessment (22). The SOFA scoring system assigns a score of 1 to 4 points to the respiratory, circulatory, renal, hematology, hepatic, and central nervous systems, depending on the extent of dysfunction.

Several studies attempted to determine if the APACHE II score was a good outcome predictor for A. baumannii bacteremia, but the results were controversial (23-25). To the best of our knowledge, the SOFA score has never been used to predict the outcome of such patients. In addition, there has not yet been an independent comparison of APACHE II and SOFA scores in patients with A. baumannii bacteremia. The aim of this study was to validate the effectiveness of the APACHE II and SOFA scoring systems in predicting the outcome of patients with A. baumannii bacteremia based on assessment scores taken at the onset of bacteremia.

\section{Patients and Methods}

\section{Patients}

This retrospective study was performed in Taipei Veterans General Hospital, a 2,900-bed tertiary medical center located in northern Taiwan. During the period of September 2004 to
December 2007, all patients with blood cultures positive for A. baumannii were included. All A. baumannii isolates were phenotypically identified by ID 32 GN (Biomerieux, St. Louis, MO) and verified by a multiplex PCR method to the genomic species level (26). For all patients, we collected data on demographics, underlying diseases, invasive procedures, clinical and microbiological data, treatment and outcomes. Information needed to calculate the SOFA and APACHE II scores at the onset of A. baumannii bacteremia were also collected. All-cause in-hospital mortality was used as the endpoint for evaluating the A. baumannii bacteremia prognosis. Since the mortality within a shorter duration may be more relevant for patients with bacterial infection, the 14day mortality was also used as another endpoint.

\section{Antimicrobial susceptibility testing}

The antimicrobial agents tested were as follows: amikacin, ampicillin/sulbactam, ceftazidime, ciprofloxacin, cefepime, colistin, gentamicin, imipenem, trimethoprim/sulfamethoxazole and piperacillin/tazobactam. The susceptibility testing of ampicillin/sulbactam, colistin, and imipenem was performed by the agar dilution method and interpreted according to the Clinical Laboratory Standards Institute (CLSI) guidelines; the tests for other antibiotics were performed by the disc diffusion method (27). Multidrugresistant A. baumannii (MDRAB) was defined when the isolate was resistant to three or more classes of the tested antimicrobial agents.

\section{Terminology}

A clinically significant $A$. baumannii bacteremia episode was defined as one in which there was one or more blood isolates of A. baumannii and clinical evidence of infection, such as fever, leukocytosis, chills, or rigors (28). The date of the first positive blood culture was considered the date of onset of A. baumannii bacteremia. A nosocomial infection was considered to be an infection that developed 48 hours or more after admission. The duration of a hospital stay was defined as the date of onset of A. baumannii bacteremia to the date of death or discharge.

A patient had end-stage renal disease (ESRD) if the creatinine clearance rate (CCR) was less than $5 \mathrm{~mL} / \mathrm{min}$ and hemodialysis was required. A patient's immunosuppressive status was considered if any of the following occurred: solid organ or stem cell transplantation, human immunodeficiency virus infection, treatment with cytotoxic chemotherapy within the previous 6 weeks, or more than 2 doses of a steroid or other immunosuppressive agent within 2 weeks of the first episode of A. baumannii bacteremia (28). Burns, recent surgeries, major trauma, or shock due to various causes were recorded if they occurred within one month of the bacteremia episode. A patient was considered to have shock (defined according to the ACCP-SCCM consensus conference) if there was evidence of organ hypoperfusion, if the systolic blood pressure was $<90$ or $>30 \mathrm{mmHg}$ relative to baseline, or if the patient needed vasopressor/inotropes to 
Table 1. Demographic and Clinical Characteristics of Patients with Acinetobacter baumannii Bacteremia

\begin{tabular}{ll}
\hline Characteristics & $\mathrm{n}=110(\%)$ \\
\hline Underlying diseases & $39(35.5)$ \\
Hypertension & $28(25.5)$ \\
Coronary artery diseases & $27(24.5)$ \\
Solid tumor & $23(20.9)$ \\
Type 2 diabetes mellitus & $20(18.2)$ \\
Previous cerebral vascular accident & $18(16.4)$ \\
Chronic obstructive pulmonary diseases & $12(10.9)$ \\
Alcoholism & $11(10)$ \\
Hematological malignancy & $10(9.1)$ \\
Congestive heart failure & $10(9.1)$ \\
Immunosuppressive status & $6(5.5)$ \\
Autoimmune diseases & $5(4.5)$ \\
End-stage renal diseases & $5(4.5)$ \\
Liver cirrhosis & $1(0.9)$ \\
Burn & $1(0.9)$ \\
Major trauma & \\
Comorbid or predisposing condition & $93(84.5)$ \\
Nosocomial infection & $73(66.4)$ \\
Central line catheterization & $70(63.6)$ \\
Mechanical ventilation & $64(58.2)$ \\
Care in intensive care unit & $42(38.2)$ \\
Recent surgeries & $38(34.5)$ \\
Steroid use & $30(27.3)$ \\
Shock & $1(0.9)$ \\
\hline Neutropenia &
\end{tabular}

maintain blood pressure despite adequate fluid resuscitation (29). Appropriate empirical antibiotics use was defined as treatment with at least one antibiotic that had in vitro activity against the pathogen and administered at the onset of A. baumannii bacteremia, with correct dosage, and with the use of at least 48 hours; otherwise, the empirical antibiotic treatment was defined as inappropriate (30). Death of a patient was considered directly related to the bacteremia if it occurred during the phase of active infection, with no evidence of another cause (31).

\section{Statistical analysis}

Descriptive data are presented as means \pm SDs. Categorical variables were compared by Fisher's exact test or the $\chi^{2}$ test, as appropriate. We assessed model calibration with the Hosmer-Lemeshow goodness-of-fit test (C statistic), which compares observed and predicted mortality. The $\chi^{2}$ is the sum of the squared differences between observed and the expected numbers, divided by the expected number $\Sigma(E-O)^{2} /$ $E$. A large value suggests poor calibration and a small value suggests good calibration (32).

Continuous variables, such as APACHE II and SOFA, were placed into classes by selecting the best cut-off points (receiver-operating characteristic analysis, ROC). Area under receiver operating characteristic curves (AUROCs) were calculated to analyze the discrimination (i.e., ability of the model to distinguish between dying and living patients) of scores using 14-day and in-hospital mortality as independent variables $(33,34)$. AUROCs between 0.7 and 0.8 were classified as "acceptable" and those between 0.8 and 0.9 as "excellent" (35).

For each ROC analysis, the cut-off values, sensitivity, specificity, overall correctness, positive prediction value (PPV), and negative prediction value (NPV) were calculated. The best cut-off point was determined when the point yielded the best specificity and sensitivity. The Youden index (36) (sensitivity + specificity -1) was calculated, and the maximum value was used to identify the optimal cut-off.

The correlation of APCHE II and SOFA was assessed by linear regression with Pearson analysis. All p-values were 2tailed, and $\mathrm{p}<0.05$ was considered to be statistically significant. The Statistical Package for the Social Sciences (SPSS) for Windows, version 17 (SPSS Inc., Chicago, IL, USA) was used for all statistical analyses.

Results

A total of 110 patients had clinically significant $A$. baumannii bacteremia during the 40-month study period. The mean patient age was $68.9( \pm 16.9)$ years and 85 patients were males. The mean APACHE II score was $24.5( \pm 10.6)$ and the mean SOFA score was $6.6( \pm 5.2)$. The mean hospital stay was $55( \pm 59)$ days, the 14-day mortality rate was $9.1 \%$, and the in-hospital mortality rate was $43.8 \%$. Table 1 shows these and additional demographics and predisposing conditions.

The antimicrobial susceptibility tests showed that colistin was the most active agent against the A. baumannii isolates (100\% susceptible), followed by imipenem (74\%), cefepime (45\%), ampicillin/sulbactam (30\%), amikacin (28\%), piperacillin/tazobactam (26\%), gentamicin (24\%), ceftazidime (22\%), ciprofloxacin (19\%), and trimethoprim/sulfamethoxazole $(17 \%)$. Ninety isolates $(82 \%)$ were MDRAB. There was no significant difference regarding the SOFA or APACHE II scores among patients infected with MDRAB or non-MDRAB. Of note, ninety-three patients $(85 \%)$ received inappropriate empirical antibiotics.

An assessment of goodness-of-fit for SOFA and APACHE II, as determined by the Lemeshow-Hosmer $\chi^{2}$ statistic, was acceptable for the 14-day and in-hospital mortality models (Table 2).

ROC analysis displays the true-positive rates (ordinate) as a function of the false-positive rates (abscissa) (Fig. 1). Calculation of the AUROC indicated that discrimination was excellent for SOFA scores and for APACHE II scores and there was no significant difference between these scores in the prediction of 14-day and in-hospital mortality (Table 3).

There was a significant correlation between SOFA and APACHE II scores $(\mathrm{p}<0.001)$ in the likelihood of 14-day and in-hospital death (Fig. 2). However, there was an exception in non-survivors within 14 days, possibly because of the small number of cases $(n=10)$.

In order to select the optimal cut-off points for predicting 14-day and in-hospital mortality, we determined the sensitiv- 
Table 2. Differences in the Calibration of SOFA and APACHE II Scores in Predicting 14-day and in-hospital Mortality for A. baumannii Bacteremia Patients

\begin{tabular}{lll}
\hline & H-L $\chi 2$ & H-L p value \\
\hline 14-day mortality & & \\
SOFA & 5.720 & 0.728 \\
APACHE II & 7.822 & 0.349 \\
\hline In-hospital mortality & & \\
SOFA & 4.045 & 0.853 \\
APACHE II & 2.441 & 0.931 \\
\hline
\end{tabular}

SOFA, Sequential Organ Failure Assessment; APACHE, Acute Physiology and Chronic Health Evaluation; H-L, Hosmer-Lemeshow C statistic.

(a)

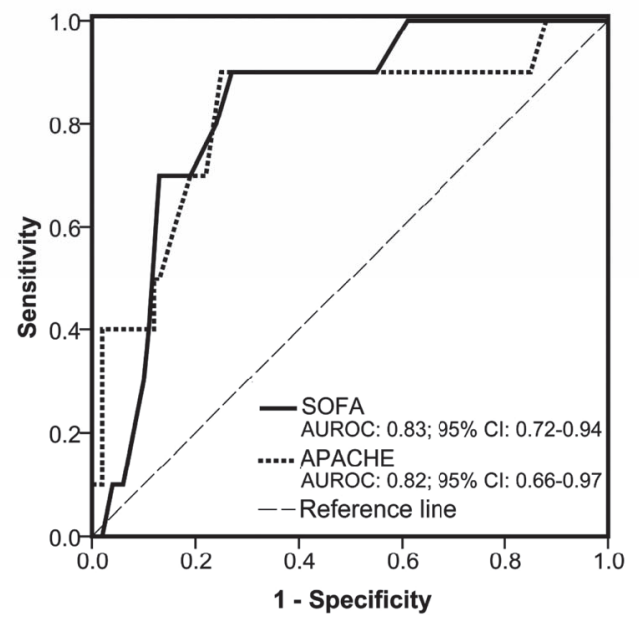

(b)

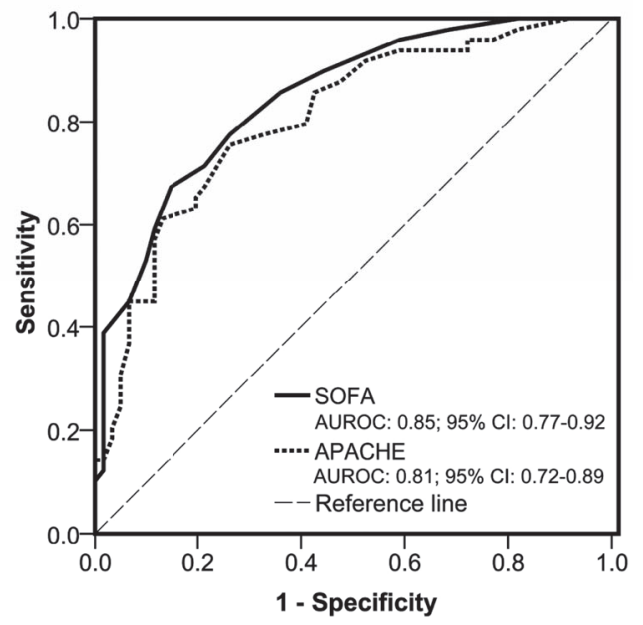

Figure 1. Receiver-operating characteristic (ROC) plot of (a) 14-day and (b) in-hospital mortality predictions using the organ failure systems for 110 A. baumannii bacteremia patients.

ity, specificity, overall correctness, PPV, NPV, and the cutoff points giving the best Youden index (Table 4). The 14day and in-hospital mortality rates differed significantly $(\mathrm{p}<$ $0.001)$ at the cut-off points of SOFA $>8$, APACHE II $>29$ and at SOFA $>7$, APACHE II $>23$ (Fig. 3). Based on the cut-off points of SOFA $>8$ and APACHE II $>29$ for prediction of 14-day mortality, both scoring systems had a high sensitivity (90\%), and high NPV (99\%), but a relatively low PPV (25-27\%). Based on the cut-off points of SOFA $>7$, and APACHE II $>23$ for the prediction of in-hospital mortality, the PPV was much higher (70-79\%).

\section{Discussion}

This study confirms that A. baumannii bacteremia is associated with a high mortality rate. In our setting, there was a 14-day mortality rate of $9.1 \%$ and an in-hospital mortality rate of $43.8 \%$, which is in general agreement with previous reports (3-8). In this study, many A. baumannii isolates were MDRAB $(82 \%)$, and many patients $(85 \%)$ received inappropriate empirical therapy, which might have been associated with the high mortality rate. Although colistin was ac- tive in vitro against all the isolates, it was not available in our hospital until year 2008 .

The present study showed that SOFA and APACHE II scores have acceptable calibration and excellent discrimination powers in predicting 14-day and in-hospital mortality. These two scores were highly correlated and equally effective in predicting mortality. This is the first study to demonstrate that the SOFA score can predict the outcome of $A$. baumannii bacteremia, and the first study to demonstrate that cut-off values of SOFA and APACHE II scores can identify patients at high risk of death from A. baumannii bacteremia. In particular, we found that SOFA $>8$ and APACHE II $>29$ was associated with higher 14-day mortality, and that SOFA $>7$ and APACHE II $>23$ was associated with higher in-hospital mortality.

In predicting 14-day mortality, both scores had high NPVs (99\%). Therefore, if a patient's SOFA score is $\leq 8$ or APACHE II $\leq 29$ at the onset of bacteremia, there is a very good likelihood that he will survive for 14 days. However, since one of the most important uses of scoring systems is to assist the physicians in critical decision-making and in explaining a patient's condition to the family, the PPV (pro- 
Table 3. Comparison of AUROCs for SOFA and APACHE II Scores in Patients with $A$. baumannii Bacteremia (Values are Means \pm SDs)

\begin{tabular}{llll}
\hline & SOFA (AUROC) & APACHE II (AUROC) & p value \\
\hline 14-day mortality & $\begin{array}{l}0.830 \pm 0.055 \\
(95 \% \text { CI: } 0.722-0.937)\end{array}$ & $\begin{array}{l}0.817 \pm 0.080 \\
(95 \% \text { CI: } 0.660-0.973)\end{array}$ & 0.887 \\
\hline In-hospital & $0.845 \pm 0.036$ & $0.806 \pm 0.042$ & 0.117 \\
mortality & $(95 \%$ CI: $0.774-0.917)$ & $(95 \%$ CI: 0.724-0.888) & \\
\hline AUROC, area under receiver operator characteristic curve; SOFA, Sequential Organ \\
Failure Assessment; APACHE II, Acute Physiology and Chronic Health Evaluation II; \\
SD, standard deviation; CI, confidence interval.
\end{tabular}

(a)

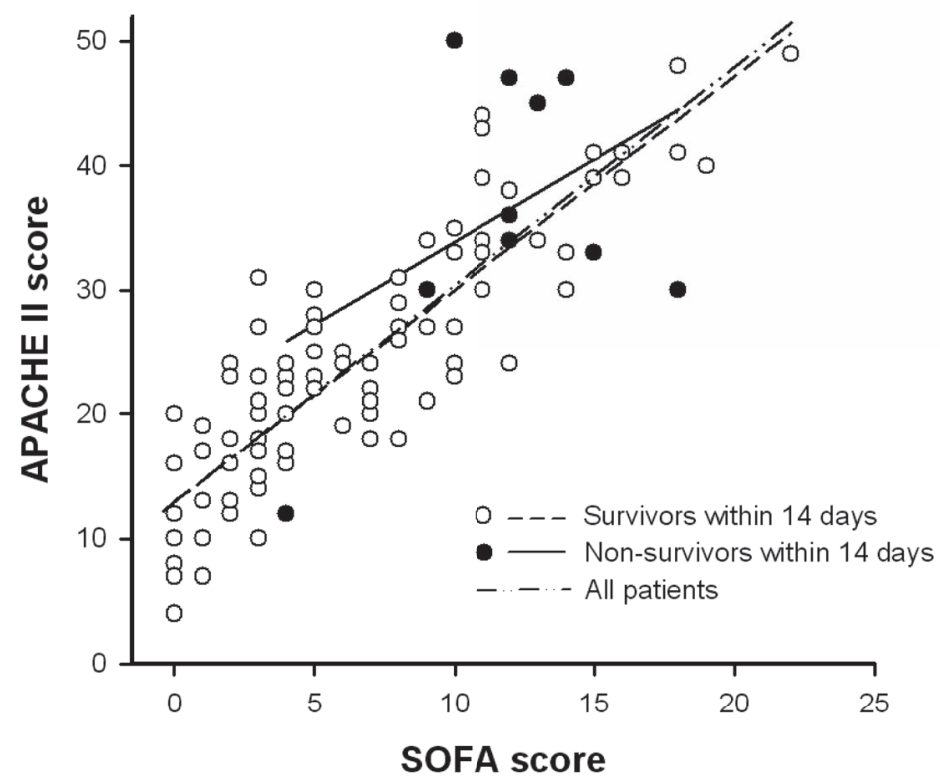

\section{Outcome}

Death

$\mathrm{y}=20.608+1.327 \mathrm{x}, \mathrm{r}=0.435$

$\mathrm{r}^{2}=0.190, \mathrm{p}=0.208$

Alive

$\mathrm{y}=12.951+1.711 \mathrm{x}, \mathrm{r}=0.869$

$\mathrm{r}^{2}=0.756, \mathrm{p}<0.001$

Total population

$y=12.972+1.750 x, r=0.851$

$\mathrm{r}^{2}=0.724, \mathrm{p}<0.001$

(b)

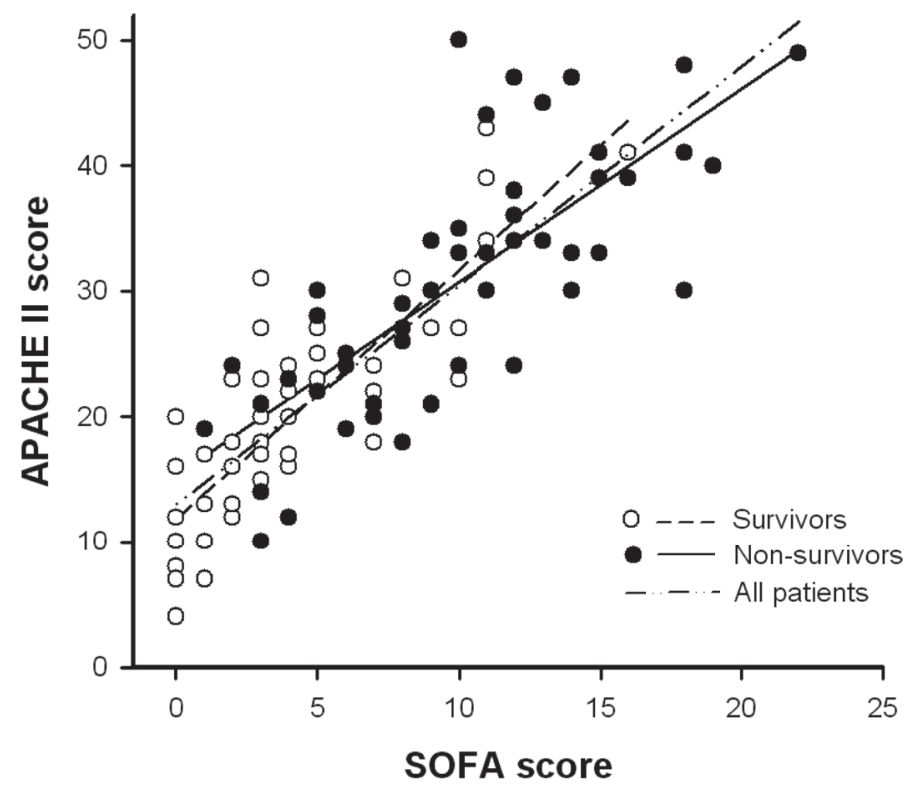

\section{Outcome}

Death

$\mathrm{y}=15.326+1.539 \mathrm{x}, \mathrm{r}=0.758$

$\mathrm{r}^{2}=0.575, \mathrm{p}<0.001$

Alive

$\mathrm{y}=11.882+1.98 \mathrm{x}, \mathrm{r}=0.836$

$\mathrm{r}^{2}=0.700, \mathrm{p}<0.001$

Total population

$\mathrm{y}=12.972+1.750 \mathrm{x}, \mathrm{r}=0.851$

$\mathrm{r}^{2}=0.724, \mathrm{p}<0.001$

Figure 2. Correlation of SOFA and APACHE II scores for (a) 14-day and (b) in-hospital mortality.

portion of patients classified as high-risk who actually succumb to disease) is probably more clinically relevant. The PPVs of SOFA and APACHE II in predicting 14-day mor- tality was only $25-27 \%$, making them poor short-term outcome predictors. However, the PPVs of SOFA and APACHE II in predicting in-hospital mortality were $70-79 \%$. Thus, 
Table 4. Prediction of Mortality at the Onset of Bacteremia

\begin{tabular}{|c|c|c|c|c|c|c|c|}
\hline & $\begin{array}{l}\text { Cut-off } \\
\text { point }\end{array}$ & $\begin{array}{l}\text { Youden } \\
\text { index }\end{array}$ & $\begin{array}{l}\text { Sensitivity } \\
(\%)\end{array}$ & $\begin{array}{l}\text { Specificity } \\
(\%)\end{array}$ & $\begin{array}{l}\text { Overall } \\
\text { correctness } \\
(\%)\end{array}$ & $\begin{array}{l}\text { PPV } \\
(\%)\end{array}$ & $\begin{array}{l}\text { NPV } \\
(\%)\end{array}$ \\
\hline \multicolumn{8}{|l|}{ 14-day mortality } \\
\hline SOFA & 8 & 0.63 & 90.0 & 73.0 & 74.5 & 25.0 & 98.6 \\
\hline APACHE II & 29 & 0.65 & 90.0 & 75.0 & 76.4 & 26.5 & 98.7 \\
\hline \multicolumn{8}{|c|}{ In-hospital mortality } \\
\hline SOFA & 7 & 0.525 & 67.3 & 85.2 & 77.3 & 78.6 & 76.5 \\
\hline APACHE II & 23 & 0.493 & 75.5 & 73.8 & 74.55 & 69.8 & 78.9 \\
\hline
\end{tabular}

(a)

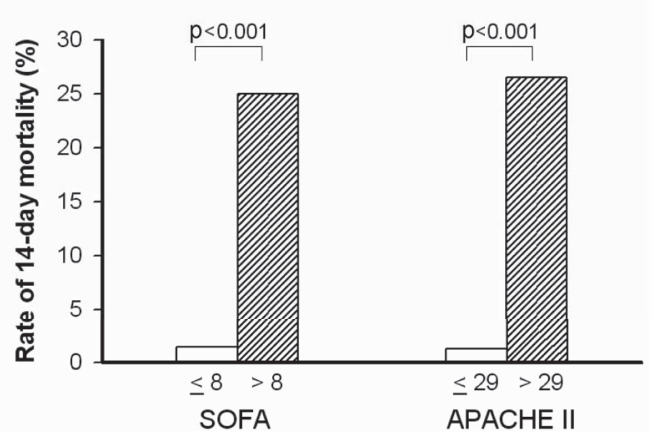

(b)

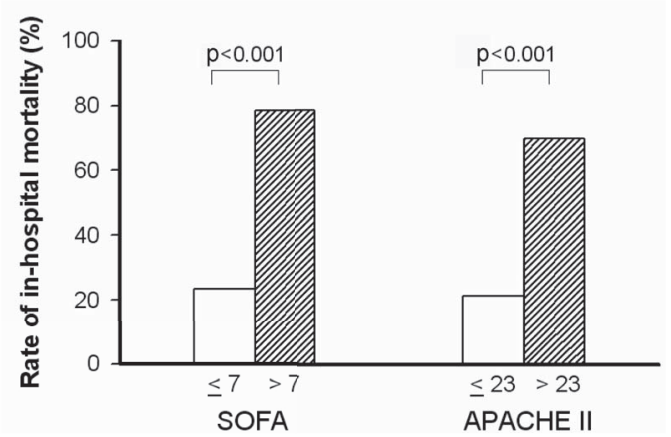

Figure 3. Mortality rates at the cut-offs giving the best Youden index for SOFA and APACHE II for (a) 14-day and (b) in-hospital mortality.

these scores may help physicians to identify patients who have a high risk of in-hospital death.

It should be noted that the present study population was relatively old (mean age of 68.9 years), predominantly male (62\%), and had relatively high SOFA and APACHE II scores at the onset of bacteremia (24). We would expect different cut-off values for other populations. Moreover, the cut-off values might be affected by other factors, such as the access to a critical care facility or the promptness of appropriate therapy. Since most of the patients in this study received inappropriate therapy at the onset of bacteremia, the roles of these two scores in the prediction of prognosis in population receiving appropriate therapy should be further validated. In addition, there are varying degrees of organ dysfunction that precede organ failure. Though the use of serial SOFA scores would seem to provide a more effective representation of the dynamics of illness (15), such calculations can be very laborious. Our study showed that SOFA and APACHE II assessed at the onset of A. baumannii bacteremia is effective for the prediction of mortality. Use of a one-time scoring system is obviously much easier for practicing clinicians.

In conclusion, this study showed that SOFA and APACHE II scores determined at the onset of A. baumannii bacteremia are reliable predictors of mortality, and that these two scores were significantly correlated. We suggest that future studies from different institutes and different geographic areas evaluate the efficacy of these scoring systems in predicting mortality from $A$. baumannii bacteremia.
The authors state that they have no Conflict of Interest (COI).

\section{Acknowledgement}

We are grateful to Miss Pui-Ching Lee for help with statistical analysis.

Funding sources: This study was supported by grant from Taipei Veterans General Hospital (V99-C1-014).

\section{References}

1. Cisneros JM, Rodriguez-Bano J. Nosocomial bacteremia due to Acinetobacter baumannii: epidemiology, clinical features and treatment. Clin Microbiol Infect 8: 687-693, 2002.

2. Eveillard M, Soltner C, Kempf M, et al. The virulence variability of different Acinetobacter baumannii strains in experimental pneumonia. J Infect 60: 154-161, 2010.

3. Seifert H, Strate A, Pulverer G. Nosocomial bacteremia due to Acinetobacter baumannii. Clinical features, epidemiology, and predictors of mortality. Medicine (Baltimore) 74: 340-349, 1995.

4. Moreno S, Vicente T, Armas M, Bernaldo de Quiros JC, Rodriguez-Creixems M, Bouza E. Nosocomial bacteremia caused by Acinetobacter. Enferm Infecc Microbiol Clin 8: 606-609, 1990.

5. Cisneros JM, Reyes MJ, Pachon J, et al. Bacteremia due to Acinetobacter baumannii: epidemiology, clinical findings, and prognostic features. Clin Infect Dis 22: 1026-1032, 1996.

6. Tilley PA, Roberts FJ. Bacteremia with Acinetobacter species: risk factors and prognosis in different clinical settings. Clin Infect Dis 18: 896-900, 1994.

7. Chiang DH, Wang CC, Kuo HY, et al. Risk factors for mortality in patients with Acinetobacter baumannii bloodstream infection with genotypic species identification. J Microbiol Immunol Infect 41: 397-402, 2008. 
8. Metan G, Sariguzel F, Sumerkan B. Factors influencing survival in patients with multi-drug-resistant Acinetobacter bacteraemia. Eur J Intern Med 20: 540-544, 2009.

9. Vincent JL, Moreno R, Takala J, et al. The SOFA (Sepsis-related Organ Failure Assessment) score to describe organ dysfunction/ failure. On behalf of the Working Group on Sepsis-Related Problems of the European Society of Intensive Care Medicine. Intensive Care Med 22: 707-710, 1996.

10. Marshall JC, Cook DJ, Christou NV, Bernard GR, Sprung CL, Sibbald WJ. Multiple organ dysfunction score: a reliable descriptor of a complex clinical outcome. Crit Care Med 23: 1638-1652, 1995.

11. Le Gall Jr, Klar J, Lemeshow S, et al. The Logistic Organ Dysfunction system. A new way to assess organ dysfunction in the intensive care unit. ICU Scoring Group. JAMA 276: 802-810, 1996.

12. Kajdacsy-Balla Amaral AC, Andrade FM, Moreno R, Artigas A, Cantraine F, Vincent JL. Use of the sequential organ failure assessment score as a severity score. Intensive Care Med 31: 243249, 2005.

13. Vincent JL, de Mendonca A, Cantraine F, et al. Use of the SOFA score to assess the incidence of organ dysfunction/failure in intensive care units: results of a multicenter, prospective study. Working group on "sepsis-related problems" of the European Society of Intensive Care Medicine. Crit Care Med 26: 1793-1800, 1998.

14. Moreno R, Vincent JL, Matos R, et al. The use of maximum SOFA score to quantify organ dysfunction/failure in intensive care. Results of a prospective, multicentre study. Working Group on Sepsis related Problems of the ESICM. Intensive Care Med 25: 686-696, 1999.

15. Ferreira FL, Bota DP, Bross A, Melot C, Vincent JL. Serial evaluation of the SOFA score to predict outcome in critically ill patients. JAMA 286: 1754-1758, 2001.

16. Zygun DA, Laupland KB, Fick GH, Sandham JD, Doig CJ. Limited ability of SOFA and MOD scores to discriminate outcome: a prospective evaluation in 1,436 patients. Can J Anaesth 52: 302308, 2005.

17. Knaus WA, Draper EA, Wagner DP, Zimmerman JE. APACHE II: a severity of disease classification system. Crit Care Med 13: 818829, 1985.

18. Oh TE, Hutchinson R, Short S, Buckley T, Lin E, Leung D. Verification of the Acute Physiology and Chronic Health Evaluation scoring system in a Hong Kong intensive care unit. Crit Care Med 21: 698-705, 1993.

19. Rowan KM, Kerr JH, Major E, McPherson K, Short A, Vessey MP. Intensive Care Society's Acute Physiology and Chronic Health Evaluation (APACHE II) study in Britain and Ireland: a prospective, multicenter, cohort study comparing two methods for predicting outcome for adult intensive care patients. Crit Care Med 22: 1392-1401, 1994.

20. Livingston BM, MacKirdy FN, Howie JC, Jones R, Norrie JD. Assessment of the performance of five intensive care scoring models within a large Scottish database. Crit Care Med 28: 18201827,2000
21. Zimmerman JE, Kramer AA, McNair DS, Malila FM. Acute Physiology and Chronic Health Evaluation (APACHE) IV: hospital mortality assessment for today's critically ill patients. Crit Care Med 34: 1297-1310, 2006.

22. Vincent JL, Ferreira F, Moreno R. Scoring systems for assessing organ dysfunction and survival. Crit Care Clin 16: 353-366, 2000.

23. Choi JY, Park YS, Kim CO, et al. Mortality risk factors of Acinetobacter baumannii bacteraemia. Intern Med J 35: 599-603, 2005.

24. Smolyakov R, Borer A, Riesenberg K, et al. Nosocomial multidrug resistant Acinetobacter baumannii bloodstream infection: risk factors and outcome with ampicillin-sulbactam treatment. J Hosp Infect 54: 32-38, 2003.

25. Brahmi N, Beji O, Abidi N, et al. Epidemiology and risk factors for colonization and infection by Acinetobacter baumannii in an ICU in Tunisia, where this pathogen is endemic. J Infect Chemother 13: 400-404, 2007.

26. Chen TL, Siu LK, Wu RC, et al. Comparison of one-tube multiplex PCR, automated ribotyping and intergenic spacer (ITS) sequencing for rapid identification of Acinetobacter baumannii. Clin Microbiol Infect 13: 801-806, 2007.

27. Clinical Laboratory Standards Institute (CLSI). Performance standards for antimicrobial susceptibility testing; Twentieth Informational Supplement M100-S20. 2010.

28. Chen HP, Chen TL, Lai CH, et al. Predictors of mortality in Acinetobacter baumannii bacteremia. J Microbiol Immunol Infect 38: $127-136,2005$

29. American College of Chest. Physicians/Society of Critical Care Medicine Consensus Conference: definitions for sepsis and organ failure and guidelines for the use of innovative therapies in sepsis. Crit Care Med 20: 864-874, 1992.

30. Camins BC, King MD, Wells JB, et al. The Impact of an antimicrobial utilization program on antimicrobial use at a large teaching hospital: a randomized controlled trial. Infect Control Hosp Epidemiol 30: 931-938, 2009.

31. Valero C, Garcia Palomo JD, Matorras P, Fernandez-Mazarrasa C, Gonzalez Fernandez C, Farinas MC. Acinetobacter bacteraemia in a teaching hospital, 1989-1998. Eur J Intern Med 12: 425-429, 2001.

32. Lemeshow S, Hosmer DW Jr. A review of goodness of fit statistics for use in the development of logistic regression models. Am J Epidemiol 115: 92-106, 1982.

33. Hanley JA, McNeil BJ. The meaning and use of the area under a receiver operating characteristic (ROC) curve. Radiology 143: 2936, 1982.

34. Hanley JA, McNeil BJ. A method of comparing the areas under receiver operating characteristic curves derived from the same cases. Radiology 148: 839-843, 1983.

35. Gursel G, Demirtas S. Value of APACHE II, SOFA and CPIS scores in predicting prognosis in patients with ventilator-associated pneumonia. Respiration 73: 503-508, 2006.

36. Youden WJ. Index for rating diagnostic tests. Cancer 3: 32-35, 1950.

(C) 2011 The Japanese Society of Internal Medicine http://www.naika.or.jp/imindex.html 